\title{
Palladium-Catalyzed Bissilylation of Arynes with Cyclic Disilanes: Synthesis of Benzo-Annulated Disilacarbocycles
}

\author{
Hiroto Yoshida,* Junnai Ikadai, Miwa Shudo, Joji Ohshita, and Atsutaka Kunai* \\ Department of Applied Chemistry, Graduate School of Engineering, Hiroshima \\ University, Higashi-Hiroshima 739-8527, Japan.
}

\section{Supporting Information}

General Remarks. All manipulations of oxygen- and moisture-sensitive materials were conducted with a standard Schlenk technique under a purified argon atmosphere. Nuclear magnetic resonance spectra were taken on a JEOL EX-270 $\left({ }^{1} \mathrm{H}, 270 \mathrm{MHz} ;{ }^{13} \mathrm{C}, 67.8 \mathrm{MHz} ;{ }^{29} \mathrm{Si}\right.$, $53.7 \mathrm{MHz})$ spectrometer or a JEOL Lambda-400 $\left({ }^{1} \mathrm{H}, 400 \mathrm{MHz} ;{ }^{13} \mathrm{C}, 99.5 \mathrm{MHz}\right)$ spectrometer using residual chloroform $\left({ }^{1} \mathrm{H}\right)$ or $\mathrm{CDCl}_{3}\left({ }^{13} \mathrm{C}\right)$ as an internal standard and tetramethylsilane $\left({ }^{29} \mathrm{Si}\right)$ as an external standard. The preparative recycling gel permeation chromatography was performed with GL Science PU 614 equipped with Shodex GPC H-2001L and -2002L columns (benzene as an eluent). Column chromatography was carried out using Merck Kieselgel 60. Unless otherwise noted, commercially available reagents were used without purification. THF was distilled from $\mathrm{Na} / \mathrm{K}$ alloy. $\mathrm{MeCN}$ was distilled from phosphorus pentoxide. 1,1,2,2tetramethyl-1,2-(1,8-naphthylene)disilane $\quad(\mathbf{1 a}),{ }^{1} \quad$ 1,1,2,2-tetramethyl-1,2-(2,2'biphenylene)disilane $\quad(\mathbf{1 b}),{ }^{2} \quad$ 1,1,2,2-tetramethyl-1,2-disilacyclopentane $\quad(\mathbf{1 c}){ }^{3} \quad{ }^{3}$ (trimethylsilyl)phenyl triflate (2a), ${ }^{4}$ 5-methyl-2-(trimethylsilyl)phenyl triflate $(\mathbf{2 b}){ }^{5} \quad{ }^{4,5-}$ dimethyl-2-(trimethylsilyl)phenyl triflate (2e), ${ }^{6} \quad$ 6-(trimethylsilyl)-5-indanyl triflate $(\mathbf{2 f}){ }^{6}{ }^{6} 3$ methoxy-2-(trimethylsilyl)phenyl triflate $(\mathbf{2 h}){ }^{7}{ }^{6}$-phenyl-2-(trimethylsilyl)phenyl triflate $(\mathbf{2 i}),{ }^{8}$ 1-(trimethylsilyl)-2-naphthyl triflate $(\mathbf{2 j}),{ }^{9}$ 3,6-dimethoxy-2-(trimethylsilyl)phenyl triflate $(\mathbf{2 l})^{6}$ and 4-methyl-2-(trimethylsilyl)phenyl triflate $(\mathbf{2 m})^{5}$ were prepared according to literature procedures. 4-Methoxy-2-(trimethylsilyl)phenyl triflate (2c), 4-phenyl-2-(trimethylsilyl)phenyl triflate (2d) and 6-methyl-2-(trimethylsilyl)phenyl triflate (2g) were prepared from 2-chloro-4methoxyphenol (Aldrich), 2-chloro-4-phenylphenol (TCI) and 2-bromo-6-methylphenol, ${ }^{10}$ respectively, in a similar manner as the preparation of 2a. 3,4-Dimethoxy-2(trimethylsilyl)phenyl triflate (2k) was synthesized from 3,4-dimethoxyphenol (Aldrich) in a similar manner as the preparation of $\mathbf{2 h}$.

Bissilylation of Arynes. A General Procedure. A THF solution $(1.0 \mathrm{~mL})$ of 1,1,3,3-tetramethylbutyl isocyanide $(0.017 \mathrm{~g}, 0.12 \mathrm{mmol}), \mathrm{Pd}(\mathrm{OAc})_{2}(1.8 \mathrm{mg}, 8.0 \mu \mathrm{mol})$ and 18 crown-6 (0.32 g, $1.2 \mathrm{mmol})$ was degassed by three freeze-thaw cycles. To this solution were added a disilane $(0.40 \mathrm{mmol})$, an aryne precursor $(0.60 \mathrm{mmol})$ and $\mathrm{KF}(0.070 \mathrm{~g}, 1.2 \mathrm{mmol})$, and 
the resulting mixture was stirred at $20{ }^{\circ} \mathrm{C}$. After the time specified in Table 1 or Scheme 2, the mixture was diluted with ethyl acetate, filtered through a Celite plug, and concentrated. Silica-gel column chromatography (hexane as an eluent) followed by gel permeation chromatography (benzene as an eluent) gave the corresponding product.

7,7,12,12-Tetramethyl-7,12-dihydro-7,12-disilapleiadene (3aa). Isolated in 66\% yield as a colorless solid: ${ }^{1} \mathrm{H}$ NMR $\left(\mathrm{CDCl}_{3}\right) \delta 0.62(\mathrm{~s}, 12 \mathrm{H}), 7.42-7.49(\mathrm{~m}, 4 \mathrm{H}), 7.75-7.77(\mathrm{~m}$, $2 \mathrm{H}), 7.86(\mathrm{dd}, J=8.2,1.2 \mathrm{~Hz}, 1 \mathrm{H}), 7.94(\mathrm{dd}, J=6.9,1.3 \mathrm{~Hz}, 1 \mathrm{H}) ;{ }^{13} \mathrm{C} \mathrm{NMR}\left(\mathrm{CDCl}_{3}\right) \delta 1.9$, 124.3, 128.3, 130.8, 133.6, 133.7, 135.2, 139.3, 140.8, 145.2; ${ }^{29} \mathrm{Si} \mathrm{NMR}\left(\mathrm{CDCl}_{3}\right) \delta$-7.4; Anal. Calcd for $\mathrm{C}_{20} \mathrm{H}_{22} \mathrm{Si}_{2}$ : C, 75.41; H, 6.96. Found: C, 75.22; H, 6.95.

7,7,9,12,12-Pentamethyl-7,12-dihydro-7,12-disilapleiadene (3ab). Isolated in 72\% yield as a colorless solid: ${ }^{1} \mathrm{H}$ NMR $\left(\mathrm{CDCl}_{3}\right) \delta 0.57(\mathrm{~s}, 6 \mathrm{H}), 0.58(\mathrm{~s}, 6 \mathrm{H}), 2.38(\mathrm{~s}, 3 \mathrm{H}), 7.22-$ $7.24(\mathrm{~m}, 1 \mathrm{H}), 7.42-7.46$ (m, $2 \mathrm{H}), 7.55$ (s, $1 \mathrm{H}), 7.64$ (d, J= 7.5 Hz, $1 \mathrm{H}), 7.83$ (d, J = 8.2 Hz, 2 $\mathrm{H}), 7.88-7.91(\mathrm{~m}, 2 \mathrm{H}) ;{ }^{13} \mathrm{C} \mathrm{NMR}\left(\mathrm{CDCl}_{3}\right) \delta 2.0,2.3,21.8,124.4,129.4,130.9,131.0,133.7$, 134.1, 134.6, 135.3, 135.4, 138.1, 139.5, 139.7, 140.9, 141.6, 145.2; ${ }^{29} \mathrm{Si} \mathrm{NMR}\left(\mathrm{CDCl}_{3}\right) \delta-7.5$, -7.4; Anal. Calcd for $\mathrm{C}_{21} \mathrm{H}_{24} \mathrm{Si}_{2}$ : C, 75.84; H, 7.27. Found: C, 75.71; H, 7.22.

9-Methoxy-7,7,12,12-tetramethyl-7,12-dihydro-7,12-disilapleiadene (3ac). Isolated in 75\% yield as a colorless solid: ${ }^{1} \mathrm{H} \mathrm{NMR}\left(\mathrm{CDCl}_{3}\right) \delta 0.60(\mathrm{~s}, 6 \mathrm{H}), 0.62(\mathrm{~s}, 6 \mathrm{H}), 3.86(\mathrm{~s}, 3 \mathrm{H})$, 6.95-6.08 (m, 1 H), 7.30-7.35 (m, 1 H), 7.38-7.51 (m, 2 H), 7.63-7.75 (m, 1 H), 7.83-7.94 (m, 4 $\mathrm{H}) ;{ }^{13} \mathrm{C} \mathrm{NMR}\left(\mathrm{CDCl}_{3}\right) \delta 1.7,2.3,54.9,113.2,120.1,124.2,124.3,130.8,131.0,133.5,135.2$, 135.3, 135.6, 135.8, 139.0, 139.6, 140.8, 147.0, 159.6; ${ }^{29} \mathrm{Si} \mathrm{NMR}\left(\mathrm{CDCl}_{3}\right) \delta$-7.6, -7.1; Anal. Calcd for $\mathrm{C}_{21} \mathrm{H}_{24} \mathrm{OSi}_{2}$ : C, 72.36; H, 6.94. Found: C, 72.31; H, 7.12.

7,7,12,12-Tetramethyl-9-phenyl-7,12-dihydro-7,12-disilapleiadene (3ad). Isolated in 54\% yield as a colorless solid: ${ }^{1} \mathrm{H}$ NMR $\left(\mathrm{CDCl}_{3}\right) \delta 0.64-0.67(\mathrm{~m}, 12 \mathrm{H}), 7.35-7.42(\mathrm{~m}, 1 \mathrm{H})$, 7.44-7.52 (m, $4 \mathrm{H})$, 7.63-7.67 (m, $3 \mathrm{H}), 7.82-7.88(\mathrm{~m}, 3 \mathrm{H}), 7.93-7.99$ (m, $3 \mathrm{H}) ;{ }^{13} \mathrm{C} \mathrm{NMR}$ $\left(\mathrm{CDCl}_{3}\right) \delta 1.9,2.0,124.3,127.2,127.3,127.4,128.8,130.95,130.97,132.5,133.6,134.3$, $135.29,135.33,139.2,139.3,140.8,141.0,141.4,143.9,145.7 ;{ }^{29} \mathrm{Si} \mathrm{NMR}\left(\mathrm{CDCl}_{3}\right) \delta-7.3$, 7.1; HRMS Calcd for $\mathrm{C}_{26} \mathrm{H}_{26} \mathrm{Si}_{2}: \mathbf{M}^{+}, 394.1573$. Found: $m / z$ 394.1569.

7,7,9,10,12,12-Hexamethyl-7,12-dihydro-7,12-disilapleiadene (3ae). Isolated in $77 \%$ yield as a colorless solid: ${ }^{1} \mathrm{H} \mathrm{NMR}\left(\mathrm{CDCl}_{3}\right) \delta 0.58(\mathrm{~s}, 12 \mathrm{H}), 2.31(\mathrm{~s}, 6 \mathrm{H}), 7.44(\mathrm{t}, J=7.5$ $\mathrm{Hz}, 2 \mathrm{H}), 7.50(\mathrm{~s}, 2 \mathrm{H}), 7.82(\mathrm{~d}, J=7.5 \mathrm{~Hz}, 2 \mathrm{H}), 7.89(\mathrm{~d}, J=7.5 \mathrm{~Hz}, 2 \mathrm{H}) ;{ }^{13} \mathrm{C} \mathrm{NMR}\left(\mathrm{CDCl}_{3}\right)$ $\delta 2.0,19.8,124.2,130.8,133.5,135.2,135.3,136.9,139.6,140.8,142.1 ;{ }^{29} \mathrm{Si} \mathrm{NMR}\left(\mathrm{CDCl}_{3}\right) \delta$ -7.7; Anal. Calcd for $\mathrm{C}_{22} \mathrm{H}_{26} \mathrm{Si}_{2}$ : C, 76.23; H, 7.56. Found: C, 76.07; H, 7.49. 
7,7,12,12-Tetramethyl-9,10-propylene-7,12-dihydro-7,12-disilapleiadene

(3af). Isolated in $76 \%$ yield as a colorless solid: ${ }^{1} \mathrm{H} \mathrm{NMR}\left(\mathrm{CDCl}_{3}\right) \delta 0.68(\mathrm{~s}, 12 \mathrm{H}), 2.15$ (quintet, $J=$ $7.5 \mathrm{~Hz}, 2 \mathrm{H}$ ), 3.04 (t, $J=7.5 \mathrm{~Hz}, 4 \mathrm{H}), 7.51$ (t, $J=7.5 \mathrm{~Hz}, 2 \mathrm{H}), 7.74$ (s, $2 \mathrm{H}), 7.89$ (d, $J=7.5$ $\mathrm{Hz}, 2 \mathrm{H}), 7.99(\mathrm{~d}, J=7.5 \mathrm{~Hz}, 2 \mathrm{H}) ;{ }^{13} \mathrm{C}$ NMR $\left(\mathrm{CDCl}_{3}\right) \delta 2.1,24.9,32.9,124.2,130.1,130.8$, 133.5, 135.1, 139.7, 140.8, 142.4, 144.7; ${ }^{29} \mathrm{Si} \mathrm{NMR}\left(\mathrm{CDCl}_{3}\right) \delta-7.3$; Anal. Calcd for $\mathrm{C}_{23} \mathrm{H}_{26} \mathrm{Si}_{2}: \mathrm{C}, 77.03 ; \mathrm{H}, 7.31$. Found: $\mathrm{C}, 76.75 ; \mathrm{H}, 7.26$.

7,7,8,12,12-Pentamethyl-7,12-dihydro-7,12-disilapleiadene (3ag). Isolated in $40 \%$ yield as a colorless solid: ${ }^{1} \mathrm{H}$ NMR $\left(\mathrm{CDCl}_{3}\right) \delta 0.50(\mathrm{~s}, 6 \mathrm{H}), 0.59(\mathrm{~s}, 6 \mathrm{H}), 2.56(\mathrm{~s}, 3 \mathrm{H}), 7.19$ $(\mathrm{d}, J=7.5 \mathrm{~Hz}, 1 \mathrm{H}), 7.29$ (t, $J=7.5 \mathrm{~Hz}, 1 \mathrm{H}), 7.42-7.48(\mathrm{~m}, 2 \mathrm{H}), 7.57(\mathrm{~d}, J=7.5 \mathrm{~Hz}, 1 \mathrm{H})$, 7.80-7.84 (m, $3 \mathrm{H}), 7.92(\mathrm{dd}, J=7.0,1.2 \mathrm{~Hz}, 1 \mathrm{H}) ;{ }^{13} \mathrm{C} \mathrm{NMR}\left(\mathrm{CDCl}_{3}\right) \delta$ 1.6, 4.6, 24.6, 124.5, 128.2, 130.2, 130.5, 131.1, 131.8, 133.1, 134.2, 135.4, 139.9, 140.2, 143.0, 143.2, 143.6, 145.8;

${ }^{29} \mathrm{Si}$ NMR $\left(\mathrm{CDCl}_{3}\right) \delta-8.2,-7.8$; Anal. Calcd for $\mathrm{C}_{21} \mathrm{H}_{24} \mathrm{Si}_{2}:$ C, 75.84; H, 7.27. Found: C, 75.63; H, 7.25.

8-Methoxy-7,7,12,12-tetramethyl-7,12-dihydro-7,12-disilapleiadene

(3ah). Isolated in $37 \%$ yield as a colorless solid: ${ }^{1} \mathrm{H} \mathrm{NMR}\left(\mathrm{CDCl}_{3}\right) \delta-0.06(\mathrm{~s}, 6 \mathrm{H}),-0.05(\mathrm{~s}, 6 \mathrm{H})$, $3.26(\mathrm{~s}, 3 \mathrm{H}), 6.35$ (d, $J=8.2 \mathrm{~Hz}, 1 \mathrm{H}), 6.68-6.93(\mathrm{~m}, 5 \mathrm{H}), 7.23-7.27$ (m, $2 \mathrm{H}), 7.42$ (dd, $J=$ 7.0, $1.2 \mathrm{~Hz}, 1 \mathrm{H}) ;{ }^{13} \mathrm{C} \mathrm{NMR}\left(\mathrm{CDCl}_{3}\right) \delta 0.3,3.1,54.9,111.0,124.4,124.5,125.6,130.0,130.1$, 130.6, 132.6, 133.2, 133.9, 135.6, 139.3, 140.4, 143.9, 146.9, 164.2; ${ }^{29} \mathrm{Si} \mathrm{NMR}\left(\mathrm{CDCl}_{3}\right) \delta-7.5$, -8.5; Anal. Calcd for $\mathrm{C}_{21} \mathrm{H}_{24} \mathrm{OSi}_{2}$ : C, 72.36; H, 6.94. Found: C, 72.10; H, 7.08.

7,7,12,12-Tetramethyl-8-phenyl-7,12-dihydro-7,12-disilapleiadene (3ai). Isolated in 30\% yield as a colorless solid: ${ }^{1} \mathrm{H}$ NMR $\left(\mathrm{CDCl}_{3}\right) \delta 0.07(\mathrm{~s}, 6 \mathrm{H}), 0.62(\mathrm{~s}, 6 \mathrm{H}), 7.03(\mathrm{t}, J=$ 7.7 Hz, $1 \mathrm{H})$, 7.24-7.33 (m, $2 \mathrm{H}), 7.36-7.51(\mathrm{~m}, 5 \mathrm{H}), 7.62$ (d, J = 8.2 Hz, $1 \mathrm{H}), 7.70-7.75$ (m, 2 $\mathrm{H}), 7.83(\mathrm{t}, J=8.1 \mathrm{~Hz}, 2 \mathrm{H}), 7.96(\mathrm{~d}, J=6.8 \mathrm{~Hz}, 1 \mathrm{H}) ;{ }^{13} \mathrm{C} \mathrm{NMR}\left(\mathrm{CDCl}_{3}\right) \delta 3.0,4.1,124.2$, 126.8, 127.1, 127.8, 128.6, 128.9, 129.2, 129.8, 130.5, 130.8, 131.4, 133.2, 133.3, 134.7, 135.4, $139.8,140.5,143.2,145.2,145.4,147.4,148.3 ;{ }^{29} \mathrm{Si} \mathrm{NMR}\left(\mathrm{CDCl}_{3}\right) \delta-8.6,-7.1$; HRMS Calcd for $\mathrm{C}_{26} \mathrm{H}_{26} \mathrm{Si}_{2}: \mathrm{M}^{+}, 394.1573$. Found: $m / z$ 394.1589.

7,7,14,14-Tetramethyl-7,14-dihydro-7,14-disilabenzo[k]pleiadene (3aj). Isolated in $33 \%$ yield as a colorless solid: ${ }^{1} \mathrm{H} \mathrm{NMR}\left(\mathrm{CDCl}_{3}\right) \delta 0.59(\mathrm{~s}, 6 \mathrm{H}), 0.76(\mathrm{~s}, 6 \mathrm{H}), 7.44-7.54$ $(\mathrm{m}, 4 \mathrm{H}), 7.80-7.88(\mathrm{~m}, 6 \mathrm{H}), 8.04(\mathrm{~d}, J=7.0 \mathrm{~Hz}, 1 \mathrm{H}), 8.31(\mathrm{~d}, J=8.2 \mathrm{~Hz}, 1 \mathrm{H}) ;{ }^{13} \mathrm{C} \mathrm{NMR}$ $\left(\mathrm{CDCl}_{3}\right) \delta 0.9,5.5,124.6,125.1,125.5,128.2,128.7,129.3,129.4,130.2,130.7,133.3,133.6$, 133.9, 135.5, 136.6, 139.6, 140.1, 143.4, 144.8, 145.0; ${ }^{29} \mathrm{Si} \mathrm{NMR}\left(\mathrm{CDCl}_{3}\right) \delta$-8.5, -7.6; Anal. Calcd for $\mathrm{C}_{24} \mathrm{H}_{24} \mathrm{Si}_{2}$ : C, 78.20; H, 6.56. Found: C, 78.19; H, 6.54. 
8,9-Dimethoxy-7,7,12,12-tetramethyl-7,12-dihydro-7,12-disilapleiadene

(3ak). Isolated in $63 \%$ yield as a colorless solid: ${ }^{1} \mathrm{H}$ NMR $\left(\mathrm{CDCl}_{3}\right) \delta 0.49(\mathrm{~s}, 6 \mathrm{H}), 0.58(\mathrm{~s}, 6 \mathrm{H}), 3.88$ (s, $3 \mathrm{H}), 3.91$ (s, $3 \mathrm{H}), 7.00$ (d, J = 8.2 Hz, $1 \mathrm{H}), 7.41-7.49$ (m, $3 \mathrm{H}), 7.80-7.83$ (m, $3 \mathrm{H})$, 7.97$8.00(\mathrm{~m}, 1 \mathrm{H}) ;{ }^{13} \mathrm{C} \mathrm{NMR}\left(\mathrm{CDCl}_{3}\right) \delta 1.0,3.5,55.3,60.7,113.2,124.37,124.44,129.9,130.2$, 130.6, 133.2, 134.1, 135.5, 136.6, 137.5, 139.6, 140.4, 143.2, 152.7, 154.1; ${ }^{29} \mathrm{Si}$ NMR $\left(\mathrm{CDCl}_{3}\right)$ $\delta$-8.6, -8.0; HRMS Calcd for $\mathrm{C}_{22} \mathrm{H}_{26} \mathrm{O}_{2} \mathrm{Si}_{2}: \mathrm{M}^{+}, 378.1471$. Found: $\mathrm{m} / z$ 378.1461.

8,11-Dimethoxy-7,7,12,12-tetramethyl-7,12-dihydro-7,12-disilapleiadene

(3al). Isolated in $72 \%$ yield as a colorless solid: ${ }^{1} \mathrm{H}$ NMR $\left(\mathrm{CDCl}_{3}\right) \delta 0.50(\mathrm{~s}, 12 \mathrm{H}), 3.78(\mathrm{~s}, 6 \mathrm{H})$, $6.87(\mathrm{~s}, 2 \mathrm{H}), 7.44(\mathrm{t}, J=7.9 \mathrm{~Hz}, 2 \mathrm{H}), 7.80(\mathrm{~d}, J=7.9 \mathrm{~Hz}, 2 \mathrm{H}), 7.91(\mathrm{~d}, J=7.9 \mathrm{~Hz}, 2 \mathrm{H}) ;{ }^{13} \mathrm{C}$ $\operatorname{NMR}\left(\mathrm{CDCl}_{3}\right) \delta 3.2,55.7,112.5,124.5,130.0,133.0,134.6,134.7,140.2,142.7,158.3 ;{ }^{29} \mathrm{Si}$ NMR $\left(\mathrm{CDCl}_{3}\right) \delta$-9.2; Anal. Calcd for $\mathrm{C}_{22} \mathrm{H}_{26} \mathrm{O}_{2} \mathrm{Si}_{2}$ : C, 69.79; H, 6.92. Found: C, 69.80; $\mathrm{H}$, 6.96.

1,1,6,6-Tetramethyl-1,6-dihydrotribenzo[b,e,g][1,4]disilocine (3ba). Isolated in $81 \%$ yield as a colorless solid: ${ }^{1} \mathrm{H}$ NMR $\left(\mathrm{CDCl}_{3}\right) \delta-0.73(\mathrm{~s}, 6 \mathrm{H}), 0.63(\mathrm{~s}, 6 \mathrm{H}), 7.32-7.36(\mathrm{~m}$, $4 \mathrm{H}), 7.39-7.44(\mathrm{~m}, 4 \mathrm{H}), 7.58-7.60(\mathrm{~m}, 2 \mathrm{H}), 7.64(\mathrm{dd}, J=5.6,3.4 \mathrm{~Hz}, 2 \mathrm{H}) ;{ }^{13} \mathrm{C}$ NMR $\left(\mathrm{CDCl}_{3}\right) \delta-0.9,0.3,126.8,128.0,128.6,129.2,134.0,135.5,137.6,146.9,151.1 ;{ }^{29} \mathrm{Si}$ NMR $\left(\mathrm{CDCl}_{3}\right) \delta$-7.8; Anal. Calcd for $\mathrm{C}_{22} \mathrm{H}_{24} \mathrm{Si}_{2}: \mathrm{C}, 76.68 ; \mathrm{H}, 7.02$. Found: $\mathrm{C}, 76.87 ; \mathrm{H}, 7.09$.

1,1,2,6,6-Pentamethyl-1,6-dihydrotribenzo[b,e,g][1,4]disilocine (3bg). Isolated in $70 \%$ yield as a colorless solid: ${ }^{1} \mathrm{H}$ NMR $\left(\mathrm{CDCl}_{3}\right)-0.76(\mathrm{~s}, 3 \mathrm{H}),-0.59(\mathrm{~s}, 3 \mathrm{H}), 0.52(\mathrm{~s}, 3 \mathrm{H})$, $0.64(\mathrm{~s}, 3 \mathrm{H}), 2.45$ (s, $3 \mathrm{H}), 7.11-7.14(\mathrm{~m}, 1 \mathrm{H}), 7.21-7.24(\mathrm{~m}, 1 \mathrm{H}), 7.31-7.36$ (m, $2 \mathrm{H})$, 7.40$7.49(\mathrm{~m}, 5 \mathrm{H}), 7.53-7.56(\mathrm{~m}, 2 \mathrm{H}) ;{ }^{13} \mathrm{C} \mathrm{NMR}\left(\mathrm{CDCl}_{3}\right)-1.4,-1.3,2.5,5.2,25.1,126.65,126.69$, 127.4, 127.5, 128.1, 129.0, 129.3, 130.6, 132.0, 135.7, 137.1, 138.9, 140.0, 143.5, 145.8, 149.2, 151.2, 151.4; ${ }^{29} \mathrm{Si} \mathrm{NMR}\left(\mathrm{CDCl}_{3}\right) \delta-8.2,-7.4$; Anal. Calcd for $\mathrm{C}_{23} \mathrm{H}_{26} \mathrm{Si}_{2}: \mathrm{C}, 77.03 ; \mathrm{H}, 7.31$. Found: C, 76.73; H, 7.39 .

2-Methoxy-1,1,6,6-tetramethyl-1,6-dihydrotribenzo[b,e,g][1,4]disilocine (3bh). Isolated in $72 \%$ yield as a colorless solid: ${ }^{1} \mathrm{H}$ NMR $\left(\mathrm{CDCl}_{3}\right)-0.76(\mathrm{~s}, 3 \mathrm{H}),-0.74(\mathrm{~s}, 3 \mathrm{H}), 0.61$ $(\mathrm{s}, 3 \mathrm{H}), 0.63(\mathrm{~s}, 3 \mathrm{H}), 3.82(\mathrm{~s}, 3 \mathrm{H}), 6.88(\mathrm{dd}, J=8.2,2.7 \mathrm{~Hz}, 1 \mathrm{H}), 7.21(\mathrm{~d}, J=2.7 \mathrm{~Hz}, 1 \mathrm{H})$, 7.32-7.45 (m, $6 \mathrm{H}), 7.59(\mathrm{~d}, J=7.9 \mathrm{~Hz}, 3 \mathrm{H}) ;{ }^{13} \mathrm{C}$ NMR $\left(\mathrm{CDCl}_{3}\right)-1.0,-0.7,0.2,0.4,54.9$, 112.4, 120.8, 126.73, 126.74, 128.5, 128.6, 129.1, 129.2, 135.4, 135.5, 135.7, 137.3, 137.4, $137.9,148.8,151.0,151.1,159.2 ;{ }^{29} \mathrm{Si} \mathrm{NMR}\left(\mathrm{CDCl}_{3}\right) \delta-8.2,-7.4 ;$ HRMS Calcd for $\mathrm{C}_{23} \mathrm{H}_{26} \mathrm{OSi}_{2}: \mathrm{M}^{+}, 374.1522$. Found: $m / 2374.1524$. 
1,1,3,6,6-Pentamethyl-1,6-dihydrotribenzo[b,e,g][1,4]disilocine (3bm). Isolated in $63 \%$ yield as a colorless solid: ${ }^{1} \mathrm{H}$ NMR $\left(\mathrm{CDCl}_{3}\right)-0.74(\mathrm{~s}, 3 \mathrm{H}),-0.73(\mathrm{~s}, 3 \mathrm{H}), 0.62(\mathrm{~s}, 3 \mathrm{H})$, 0.64 (s, $3 \mathrm{H}), 2.35$ (s, $3 \mathrm{H}), 7.17$ (d, $J=7.7 \mathrm{~Hz}, 1 \mathrm{H}), 7.32-7.36(\mathrm{~m}, 2 \mathrm{H}), 7.39-7.42$ (m, $4 \mathrm{H})$, $7.46(\mathrm{~s}, 1 \mathrm{H}), 7.54-7.60(\mathrm{~m}, 3 \mathrm{H}) ;{ }^{13} \mathrm{C} \mathrm{NMR}\left(\mathrm{CDCl}_{3}\right)-0.9,-0.8,0.3,21.5,126.72,126.74$, $128.5,128.6,128.9,129.1,129.2$, 134.2, 135.0, 135.4, 135.5, 137.56, 137.64, 137.8, 143.2, 146.8, 151.08, 151.13; ${ }^{29} \mathrm{Si} \mathrm{NMR}\left(\mathrm{CDCl}_{3}\right) \delta-8.1,-7.9$; Anal. Calcd for $\mathrm{C}_{23} \mathrm{H}_{26} \mathrm{Si}_{2}$ : C, 77.03; H, 7.31. Found: C, 76.83; H, 7.32.

\section{1,1,6,6-Tetramethyl-6,7,8,9-tetrahydro-(1H)-benzo[b][1,4]disilepine}

(3ca). Isolated in 55\% yield as a colorless oil: ${ }^{1} \mathrm{H} \mathrm{NMR}\left(\mathrm{CDCl}_{3}\right) 0.27(\mathrm{~s}, 12 \mathrm{H}), 0.99(\mathrm{t}, J=6.5 \mathrm{~Hz}, 4$ H), 1.91 (quintet, $J=6.5 \mathrm{~Hz}, 2 \mathrm{H}$ ), 7.32 (dd, $J=5.5,3.2 \mathrm{~Hz}, 2 \mathrm{H}$ ), 7.59 (dd, $J=5.5,3.2 \mathrm{~Hz}, 2$ $\mathrm{H}) ;{ }^{13} \mathrm{C} \mathrm{NMR}\left(\mathrm{CDCl}_{3}\right)-0.3,18.0,31.6,127.7,134.4,146.9 ;{ }^{29} \mathrm{Si} \mathrm{NMR}\left(\mathrm{CDCl}_{3}\right) \delta-0.8$; Anal. Calcd for $\mathrm{C}_{13} \mathrm{H}_{22} \mathrm{Si}_{2}$ : C, 66.59; H, 9.46. Found: C, 66.45; H, 9.30.

\section{References}

(1) Kiely, J. S.; Boudjouk, P. J. Orgnomet. Chem. 1979, 182, 173-183.

(2) Kira, M.; Sakamoto, K.; Sakurai, H. J. Am. Chem. Soc. 1983, 105, 7469-7470.

(3) Kumada, M.; Tamao, K.; Takubo, T.; Ishikawa, M. J. Organomet. Chem. 1967, 9, $43-55$.

(4) Himeshima, Y.; Sonoda, T.; Kobayashi, H. Chem. Lett. 1983, 1211-1214.

(5) Yoshikawa, E.; Radhakrishnan, K. V.; Yamamoto, Y. J. Am. Chem. Soc. 2000, 122, $7280-7286$.

(6) Yoshida, H.; Sugiura, S.; Kunai, A. Org. Lett. 2002, 4, 2767-2769.

(7) Peña, D.; Pérez, D.; Guitián, E.; Castedo, L. J. Am. Chem. Soc. 1999, 121, 5827-5828.

(8) Yoshida, H.; Shirakawa, E.; Honda. Y.; Hiyama, T. Angew. Chem. Int. Ed. 2002, 41, $3247-3249$.

(9) Peña, D.; Pérez, D.; Guitián, E.; Castedo, L. J. Org. Chem. 2000, 65, 6944-6950.

(10) Knölker, H. -J.; Fröhner, W.; Wagner, A. Tetrahedron Lett. 1998, 39, 2947-2950. 